\title{
LIGHT FROM DARK CLOUDS
}

\author{
Joachim A. Stüwe \\ Astronomisches Institut der Ruhr-Universität \\ Bochum, Postfach 102148 \\ D-4630 Bochum 1 \\ FRG
}

\section{INTRODUCTION}

The purpose of the present investigation was to find out how far the existing photographic sky surveys can be used to derive physical parameters of dark clouds, without the need of new observations. In a recent paper a method of distance determination and extinction mapping of dark clouds by automated star counts on microphotometric raster scans of the ESO/SRC Southern Sky Survey (Stüwe, 1989, hereafter Paper I) is presented. The present paper does a second step, which is based on the fact that dark clouds are marked by an intrinsic surface brightness (Barnard, 1927).

\section{MATERIALS AND METHODS}

Microphotometric scans of some dark clouds were used to determine the total surface brightness in the direction of the clouds by the use of an algorithm developed by C.P. de Vries (1986). In areas typically larger than the image of an average star, a histogram of the photographic density is computed. To the lower part of this histogram, which is less influenced by stars, a Gaussian is fitted, which gives the value of the photographic density corresponding to the surface brightness. From this value a map of the surface brightness in arbitrary flux units is obtained by applying the calibration curves given by the gray wedges on each survey plate. Figure 1 shows an example of this approach. Part I shows the original photograph of a dark cloud. In Part II the extinction map of this region is shown, where the grey scales represent the extinction values ranging from $0 \mathrm{~m} 0$ (white) to $3 \mathrm{~m} .0$ (black). Part III shows the resultant surface brightness distribution, which is still contaminated by the brightest stars in the field, but nevertheless clearly shows the dark core (white) of the cloud surrounded by a feeble bright rim (light grey).

\section{RESULTS AND DISCUSSION}

The blue (SRC-J) photographs of two clouds were further investigated and the main results are listed in Table 1. To estimate the physical units of the surface brightness, the relative intensities were calibrated using the flux predicted by the surface photometry of the Milky Way (Kimeswenger, et al., 1989, this volume), in the appropriate directions. The quoted $S_{10}(B)$ values then have an estimated error in the zero point of about $20 \%$ whereas the intrinsic error is unaltered and is less than $10 \%$. Figure 2 shows the mean radial profiles of the observed surface brightness as dashed lines. The solid lines represent free hand fits to this distribution ignoring contamination due to bright foreground stars. From these plots the values of the average surrounding sky brightness $I_{\text {obs }}\left(\right.$ sky), the brightness of the rim $I_{o b s}($ rim $)$, and the brightness 


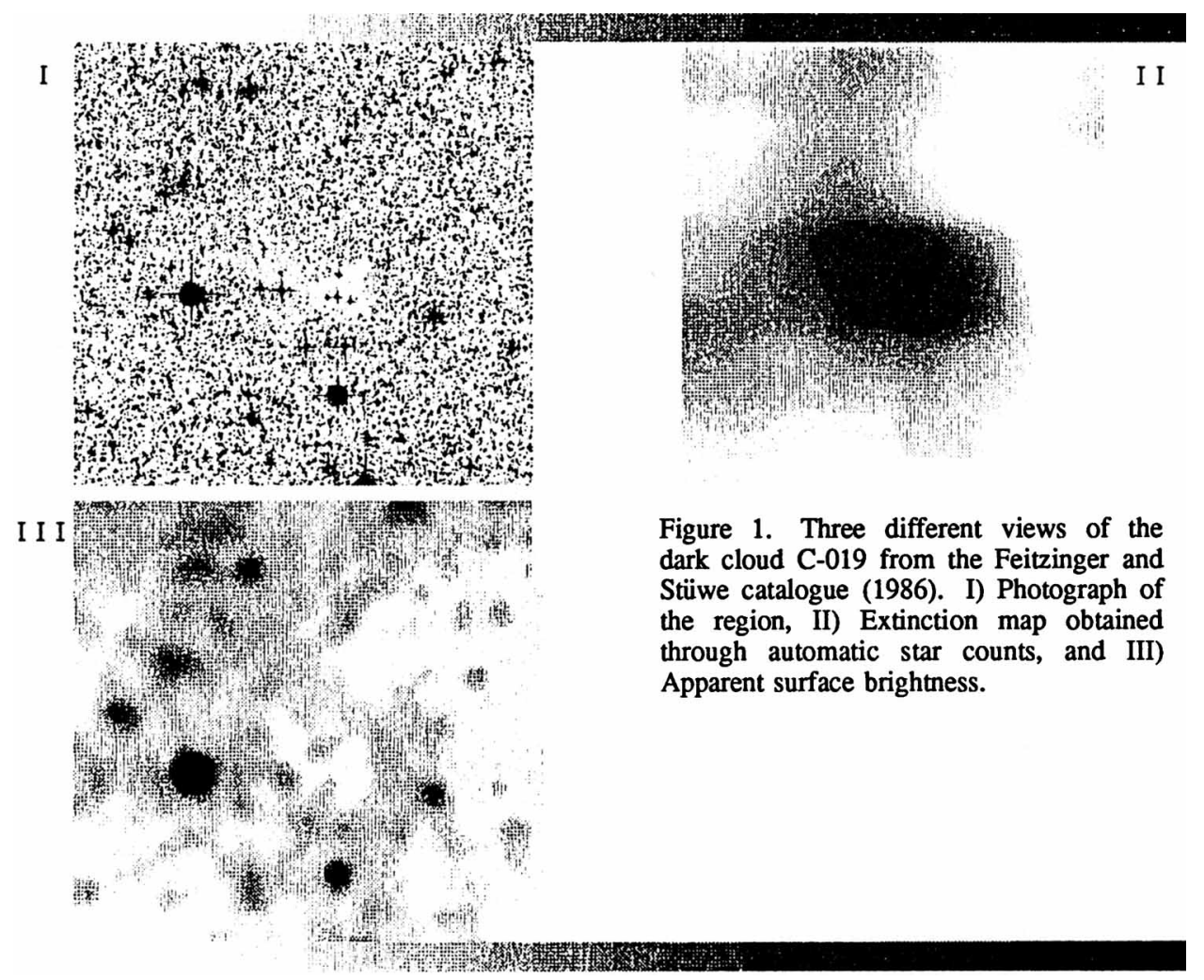

of the core $I_{\text {obs }}$ (core) in Table 1 are obtained, whereas the values for the distance, the extinction data and the radius of the dark clouds are taken from Paper I. The observed flux can be considered as the sum of the three components $I_{f g}, I_{b g}$ and $I_{s c a}$

$$
I_{o b s}=I_{f g}+I_{b g} * 10^{-0.4 A_{B}}+I_{s c a} * 10^{-0.4 A_{f g}} \text {. }
$$
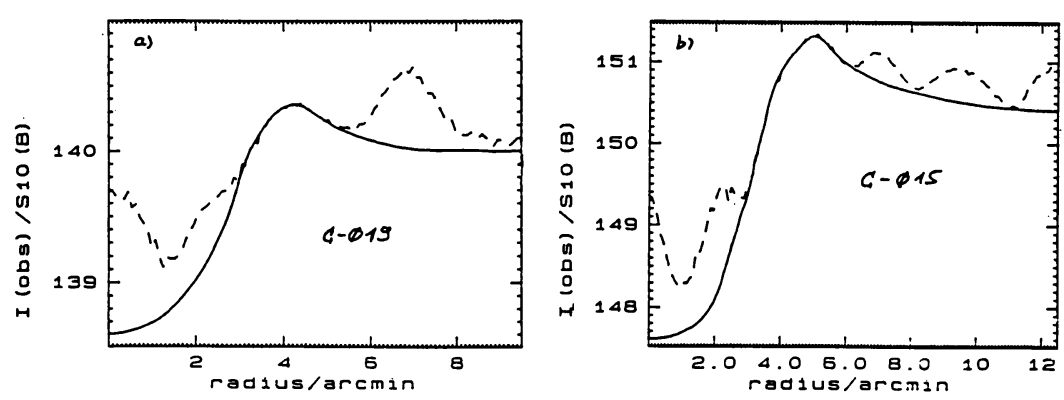

Figure 2. Mean radial profiles of the apparent surface brightness, a) C-019 and b) C-015.

The foreground extinction and the extinction distribution are known from Paper I. The foreground extinction may not be neglected because of the rather large distances of the clouds 
(Table 1), but in the difference between the core value $I_{\text {obs }}$ (core) and the average sky brightness $I_{o b s}(s k y)$, the foreground value cancels out. The assumption that the apparent brightness of the scattered light coming from the direction of the cloud core is roughly the same or even smaller than the brightness of the rim above the sky is confirmed by various model calculations (Mattila, 1970b, 1979; Witt \& Stephens, 1974; FitzGerald, Stephens, and Witt, 1976). This leads to a reasonable estimate of the background brightness (Table 1). Now the absolute flux of the light scattered by the dust particles in the cloud can be computed

$$
I_{s c a}=\left[I_{o b s}-I_{o b s}(s k y)-I_{b g} *\left(10^{-0.4\left(A_{f g}+A_{c o r e}\right)}-10^{-0.4 A_{f g}}\right)\right]
$$

The resulting mean radial profiles are shown in Figure 3 and may directly be compared with appropriate model calculations.

TABLE 1. Results of the surface brightness determination.

The values for $d, A_{f g}, A_{\text {core }}$ and $R_{d . c .}$ are taken from Stüwe (1989)

\begin{tabular}{ccccccccccc}
\hline \hline cloud & 1 & $b$ & $d / p c$ & $\frac{R_{\text {rim }}}{R_{d c .}}$ & $A_{f g}$ & $A_{\text {core }}$ & $\begin{array}{c}I_{\text {obs }} \\
(\text { sky })\end{array}$ & $\begin{array}{c}I_{\text {obs }} \\
(\text { rim })\end{array}$ & $\begin{array}{c}I_{\text {obs }} \\
(\text { core })\end{array}$ & $I_{b g}$ \\
\hline $\mathrm{C}-019$ & 251.0 & -1.4 & 800 & 1.0 & 0.7 & 2.4 & $140.0 S_{10}$ & $140.3 S_{10}$ & $138.6 S_{10}$ & $5 S_{10}$ \\
$\mathrm{C}-015$ & 249.0 & -3.2 & 500 & 0.8 & 2.5 & 1.7 & $150.4 S_{10}$ & $151.3 S_{10}$ & $147.6 S_{10}$ & $50 S_{10}$ \\
\hline
\end{tabular}

Extinction measurements yield information about the distances, sizes and masses of dark clouds (Stüwe, 1989). Additionally, the analysis of the surface brightness distribution yields clues on the albedo, $a$, and the phase function asymmetry, $g$, of the scattering particles (Mattila, 1970b, 1979) and on the internal density structure of the dark clouds (Witt \& Stephens, 1974). Although the models in the literature are not directly comparable to the data presented here, a comparison at least yields clues to the correct parameters. For a detailed analysis, appropriate model calculations are indispensable. Figure 4 suggests an albedo of $a=0.6$ for an adopted $g=0.75$. The relatively large ratios between the radius of the bright rim $R_{\text {rim }}$ and the dark clouds $R_{d c}$ indicate the presence of an internal density law $n \sim r^{-\beta}$ in the clouds, with an exponent $\beta<2$. This is consistent with the value obtained from the extinction measurements of $\beta \approx 1.3$ (Stüwe, 1989). The low background value for cloud C-019 is consistent with model fits to the observed surface brightness of the Milky Way (Kimeswenger, et al., 1989, this volume), which predicts $95 \%$ of the observed brightness in this direction to originate at distances closer than $1 \mathrm{kpc}$. The values for $\mathrm{C}-015$ also fit into this picture so that the surface brightness meas-

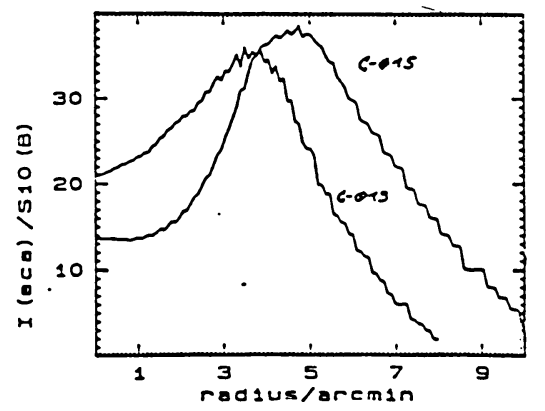

Figure 3. Mean radial profiles of the light scattered by dust grains in the dark clouds. (The values of C-019 have been multiplied by 10.)

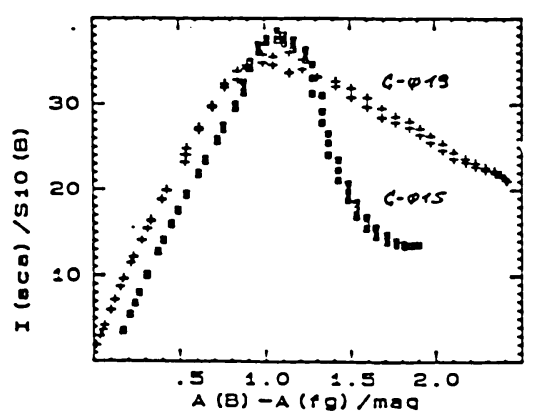

Figure 4. Scattered light versus optical depth $\left(-A_{B}-A_{f g}\right)$. (The values for C-019 have been multiplied by 10 .) 
urements yield an independent confirmation of the distances derived from star counts.

The reduction procedure presented together with that of a recent investigation (Stüwe, 1989) leads to the conclusion that Schmidt survey photographs are well suited for studies of the physical properties of dark clouds. The present photographic sky surveys analysed by image processing techniques may help to improve our knowledge of that part of the interstellar medium manifested in dark clouds.

\section{REFERENCES}

Barnard, E.E. 1927, "Atlas of Selected Regions of the Milky Way," ed. E.B. Frost and M.R. Calvert (Carnegie Institution, Washington).

Kimeswenger, S., Hoffmann, B., Schlosser, W., Schmidt-Kaler, Th. and Seidensticker, K.J. 1989, Proc. IAU 139, Galactic and Extragalactic Background Radiation, ed. S. Bowyer, and Ch. Leinert (Dordrecht: Kluwer Academic Publishers).

Feitzinger, J.V. and Stüwe, J.A. 1984, Astron. and Astrophys. Suppl. Ser. 58, 365.

FitzGerald, M.P., Stephens, T.C. and Witt, A.N. 1976, Astrophys. J. 208, 709.

Mattila, K. 1970a, Astron. and Astrophys. 8, 273.

Mattila, K. 1970b, Astron. and Astrophys. 9, 53.

Mattila, K. 1979, Astron. and Astrophys. 78, 253.

Stïwe, J.A. 1989, Astron. and Astrophys., submitted (Paper I).

Vries, C.P. de 1986, Ph.d. thesis (Sterrewacht Leiden, The Netherlands).

Witt, A.N. and Stephens, T.C. 1974, Astron. J. 79, 948.

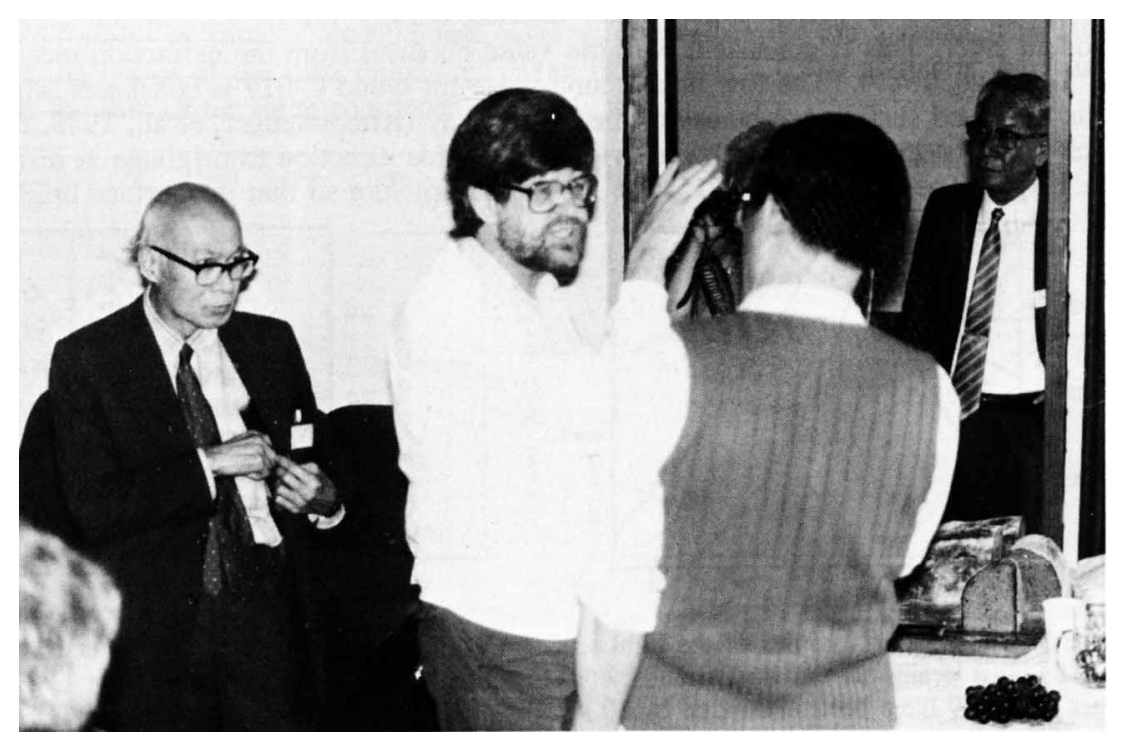

Ko Aizu, Gerrit Verschuur, Yaoquan Chu, and Hiroyoshi Tanabe 